\section{Percepção de gestantes do Programa Saúde da Família em relação a barreiras no atendimento odontológico em Pernambuco, Brasil}

\author{
Pregnant women's perceptions of the Family \\ Health Program concerning barriers to dental \\ care in Pernambuco, Brazil
}

Olga Maria Ramalho de Albuquerque 1 Claídes Abegg 2 Cecile Soriano Rodrigues 3

\title{
Introdução
}

1 Universidade de Pernambuco, Recife, Brasil.

2 Programa de Pós-graduação em Saúde Coletiva

Universidade Luterana do Brasil, Canoas, Brasil.

3 Programa de Pós-graduação em Odontologia, Universidade de Pernambuco, Camaragibe, Brasil.

\section{Correspondência} Olga Maria Ramalho de Albuquerque

Av. Gov. Agamenon Magalhães 1500/802, Recife, PE 52030-210, Brasil. olgamaria@terra.com.br

\begin{abstract}
This study focused on barriers to dental care for pregnant women registered with the Health Family Program in Cabo de Santo Agostinho, Pernambuco, Brazil. A qualitative approach using three focal groups was adopted. Each focal group included four to nine pregnant women. The information was analyzed by means of content analysis. The main individual barriers were folk beliefs that discouraged dental care during pregnancy, lack of perceived need, and fear of pain. In addition, the women reported difficulties in leaving home in the early hours of the morning to meet a dental appointment, highlighting an aspect of barriers to dental care which has not been identified in other studies. The article concludes by emphasizing the importance of health education for pregnant women, humanization of dental care, and continuing education for health professionals.
\end{abstract}

Pregnant Women; Family Health Program; Dental Health Services
Existem poucas informações acerca do uso de serviços odontológicos por parte da população brasileira. Conforme dados da Pesquisa Nacional por Amostragem de Domicílios 1, quase um quinto $(18,7 \%)$ da população brasileira nunca consultou um dentista. As maiores proporções de pessoas desassistidas estão entre as crianças menores de quatro anos $(85,6 \%)$, nos homens $(20,5 \%)$ e na população residente em áreas rurais $(32,0 \%)$.

Provavelmente incluídas nesta porcentagem de pessoas que não procuram o dentista, estão as que enfrentam barreiras ao atendimento odontológico, conceituadas por Freeman 2 como aquelas dificuldades que as pessoas vivenciam para obter atendimento em odontologia.

As barreiras ao atendimento odontológico identificadas por Freidson \& Feldman 3 , e posteriormente por Kegeles 4, foram relacionadas ao medo e à ansiedade. Em outro estudo, Gale 5 argumentou que o medo estava associado à desaprovação do dentista. Alem disso, Kleinknecht et al. 6 sugeriram que o medo se origina de experiências negativas anteriores, bem como de características pessoais e profissionais do dentista.

No documento intitulado Melhorando o Acesso à Saúde Bucal, as barreiras ao atendimento odontológico foram classificadas em três categorias: relacionadas ao indivíduo, à prática da profissão e à sociedade 7 . 
As barreiras concernentes ao indivíduo são: baixa percepção de necessidade, ansiedade e medo, custos e dificuldade de acesso. Entre os fatores limitantes à prática da odontologia figuram a inadequação dos recursos humanos, a formação inadequada dos profissionais para contribuir na mudança das condições de saúde da população, a má distribuição geográfica da mão-de-obra e a ocorrência de estresse ocupacional. As barreiras concernentes à sociedade se referem ao número insuficiente de ações de promoção de saúde, a instalações impróprias dos serviços e ao reduzido apoio financeiro à pesquisa 7 .

Embora não exista um consenso na discussão acerca da periodicidade da visita ao dentista $8,9,10,11,12$, para as gestantes, a procura do atendimento torna-se um meio de intensificar procedimentos preventivos de modo a evitar doenças bucais associadas à gravidez 13,14,15,16.

Estudos têm demonstrado que gestantes vão menos ao dentista que mulheres de grupo etário semelhante 17,18 .

No Cabo de Santo Agostinho, Pernambuco, Brasil, os mapas de produtividade do serviço odontológico dirigido a gestantes foram avaliados no período de julho de 1999 a junho de 2000. A discrepância entre o número de gestantes agendadas e aquelas que, efetivamente, compareceram ao agendamento variou de 8,0 a $36,0 \%$, com média de $24,5 \%$. Esses resultados evidenciam a presença de barreiras que vão além do acesso propriamente dito 19 .

A importância da identificação das barreiras ao atendimento odontológico responde à necessidade de atingir clientelas específicas e sondar grupos ainda não alcançados pelos serviços de saúde, como gestantes, por exemplo. Tais grupos, em geral, não buscam esses serviços por iniciativa própria porque embora percebam as necessidades de tratamento, não se sentem seguros quanto à sua indicação formal 20.

Ao investigar barreiras ao atendimento odontológico, salientam-se questões fundamentais que dizem respeito à eqüidade, integralidade, humanização, acesso e qualidade da atenção odontológica. As principais questões levantadas neste estudo são: (a) existem barreiras ao atendimento odontológico de gestantes no Cabo de Santo Agostinho?; e (b) qual a natureza dessas barreiras?

O presente trabalho teve como objetivo identificar e analisar qualitativamente barreiras individuais ao atendimento odontológico de gestantes cadastradas no Programa Saúde da Família (PSF) do Cabo de Santo Agostinho.

\section{Método}

A abordagem do presente estudo é qualitativa, utilizando-se a técnica dos grupos focais. A abordagem qualitativa favorece a identificação em detalhes das atitudes, necessidades e valores de grupos-alvo específicos, além da avaliação de programas de educação em saúde e de políticas sociais voltadas para a saúde bucal 21 Tais avaliações demandam a compreensão mais profunda de opiniões, significados, emoções, ações e o modo como tudo isso afeta o comportamento em saúde no ambiente onde a pessoa vive 22,23 .

Com o uso da pesquisa qualitativa é possível aprofundar o conhecimento em estágio precoce da pesquisa 23 , investigar questões sociológicas relacionadas à saúde 22 , entender comportamentos não aparentes, além de revelar de maneira acurada e com bases científicas o pensamento das pessoas sobre a atenção em saúde bucal e as visitas ao consultório odontológico ${ }^{21}$.

Diferentes estudos qualitativos adotaram a discussão em pequenos grupos, grupos focais, entre eles Blinkhorn et al. 24, Nettleton 22, Finch et al. 20, Redford \& Gift 25. Segundo Krueger 26, os grupos focais reúnem pessoas com determinadas características para gerar dados de natureza qualitativa mediante uma discussão com enfoque pré-definido.

O grupo de gestantes que participou do estudo foi selecionado de forma intencional, já que o objetivo foi examinar a natureza e a variedade do fenômeno 23,27,28,29. Por essa razão, as características requeridas para inclusão nos grupos focais foram: morar no Cabo de Santo Agostinho, ser inscrita no PSF, fazer o pré-natal, estar no terceiro trimestre gestacional, não ter procurado o serviço de odontologia durante a gravidez e partilhar elementos comuns de vivência social.

O PSF se caracteriza por ser uma estratégia de atenção básica que tem entre seus objetivos a ampliação de acesso aos serviços de saúde. No Cabo de Santo Agostinho, cerca de $78,0 \%$ da população é acompanhada pelas vinte e seis equipes do PSF. $\mathrm{O}$ atendimento odontológico das gestantes é realizado num consultório destinado a esta clientela, instalado em um dos centros onde se realiza o pré-natal, para facilitar $o$ acesso mediante agendamento.

No processo de identificação e nas discussões com os grupos focais, vale salientar a contribuição das agentes comunitárias de saúde no terceiro trimestre gestacional, incluídas nos critérios de seleção para participar de um dos grupos focais. 
Os dados foram coletados em discussões com três grupos focais e um "brainstorm" com a participação de quatro a nove diferentes gestantes em cada grupo focal. Utilizou-se um questionário não estruturado. As discussões nos grupos focais foram gravadas e posteriormente transcritas. Os dados foram analisados com o uso da análise de conteúdo, orientada pelo plano de pesquisa e pelos objetivos do estudo, adotando-se o tema como regra de recorte e tomando-se por base a unidade de registro 30 .

O projeto desta pesquisa foi aprovado pelo Comitê de Ética e Pesquisa do Centro de Ciências da Saúde da Universidade Federal de Pernambuco sob o protocolo no 132/2000.

\section{Resultados e discussão}

Identificação e análise das barreiras ao atendimento odontológico de gestantes do Cabo de Santo Agostinho

Segundo o Programa de Desenvolvimento da Organização das Nações Unidas 31 , no período de 1970 a 1991, o Índice de Condições de Vidaeducação do Cabo de Santo Agostinho cresceu 83,5\%, enquanto o Índice de Condições de Vida-renda aumentou apenas em 27,7\%. É nesse contexto que se situa o presente trabalho, no qual as gestantes mencionam barreiras peculiares à sua condição social de moradia e de condições de vida.

\section{Barreiras referentes ao indivíduo}

\section{- Baixa percepção de necessidade}

As reuniões com os grupos focais identificaram os componentes desse domínio, entre os quais a falta de interesse, a preguiça, o comodismo, o esquecimento, a indiferença, o fato de não gostar de dentista ou de nem pensar em ir ao dentista durante a gravidez. Além destes, foram mencionadas também a baixa valorização da saúde bucal, a pouca importância atribuída aos dentes, a baixa apreciação dos resultados do tratamento, as crenças populares que desaconselham a ir ao dentista no período gestacional.

Este elenco de barreiras concernentes ao indivíduo também foram identificadas por Finch et al. 20, Rogers 17, Tomar et al. 32, Jandrey \& Drehmer 33, Lahti et al. 34 e Nuto \& Nations 35.

Nos diferentes grupos focais, gestantes primíparas e multíparas na terceira, quinta e nona gravidez referem não ter ido ao dentista porque "não precisou" ou "nem pensei em ir ao dentista", mesmo tendo sentido dor de dente durante a gravidez, caracterizando a baixa percepção de necessidade. Algumas delas chegaram a associar a dor de dente ao estado gestacional “...é normal quando a pessoa está grávida ter dor de dente... até o dente bom fica doendo...".

Verificou-se a existência de um consenso entre as gestantes, em todos os grupos focais, quando questionadas acerca dos comentários ouvidos das pessoas sobre ir ao dentista na gravidez. São relatos de familiares, de amigos e até mesmo de profissionais de saúde que vão passando como uma herança cultural fortemente disseminada “...diz que não pode de jeito nenhum, pode dar hemorragia." "Tem gente que até fala assim: 'se a pessoa quiser perder um filho, arranque um dente.'”

"Enquanto o dente não incomodou eu não fui..." ou "...o que empurra para ir [ao dentista] é uma dor de dente...”. Esta opinião era compartilhada por gestantes de outros grupos. A reação geral hilariante observada entre as participantes, sugere uma atitude de assentimento tácito, quase uma revelação da indiferença em relação ao estado de saúde da boca, que a frase inacabada deixa subentendida.

"Fui levando, fui levando, botei uma coisa, outra [no dente], depois senti tanta dor de dente que quase enlouqueci...", essa gestante é agente de saúde, com acesso ao atendimento odontológico, independente de marcação de consulta. A expressão usada "fui levando, fui levando" dá idéia de permanência, sugere comodismo como atesta outra declaração sua, "eu fui [ao dentista], mas eu 'tava alucinada de dor"', que mostra uma tomada de consciência tardia despertada por uma dor de dente forte e contínua.

Por um lado verifica-se a existência de barreiras referentes ao indivíduo, representadas pela baixa percepção de necessidade. Por outro lado, a dificuldade representada pela crença cultural que desaconselha o tratamento odontológico durante a gravidez “...não pode, não deve extrair o dente, a mulher não pode tomar anestesia, não pode fazer tratamento, pra pessoa esse negócio de boca na gravidez é um mito...". A conjugação das barreiras gera uma apatia, revelada pela conformação ao que foi estabelecido pela cultura dominante.

“Não, eu nunca procurei o dentista durante a gravidez não. Na época que eu estava grávida do meu segundo menino, senti muita dor dente, fiquei quase os nove meses [sofrendo]... depois que ele nasceu, eu descuidei... Depois de quatro meses engravidei de novo e dessa vez tenho dois dentes que dói... mas todo mundo fala que não posso extrair porque estou grávida. O outro [dente] está bom, mas ele dói também”. Essa 
opinião revela que a irreversibilidade e a cronicidade dos processos destrutivos como cárie e doença periodontal e sua relação com a saúde geral não são levados em conta pelas gestantes ao decidir procurar o dentista, mas apenas a agudização de processos inflamatórios.

Ao mesmo tempo que os depoimentos indicam baixa percepção de necessidade, revelam também a inexistência de um objetivo comum entre o usuário, que busca o atendimento, e o dentista, que presta o serviço. “...não quer obturar porque [a restauração] cai, dói, porque extraindo, não vai mais sentir dor, não vai voltar mais ao dentista". Na forma de expressão da gestante está implícita a baixa apreciação dos resultados do tratamento, a distância existente entre os dois lados de um processo de participação mútua no qual dentista e paciente deveriam trabalhar para alcançar a saúde.

\section{- Ansiedade e medo}

Entre as fontes de ansiedade e medo percebidas pelas gestantes estudadas estão a possibilidade de sentir dor e desconforto; de reprimenda por parte do dentista; do barulho da turbina; da anestesia; dos instrumentos utilizados; de embaraço; da sala fria; de desconforto com aspectos sensitivos como cheiro, sabor, visão dos instrumentos, refletor, uniformes, máscaras, posição da cadeira na horizontal.

Estes aspectos que representam barreiras individuais ao atendimento odontológico foram também identificadas por Galé 5 , Rogers 17, Bernd et al. 36, Tomar et al. 32 , Lahti et al. 34 e Nuto \& Nations 35.

Desde a primeira reunião com as gestantes, as mais recorrentes associações apontadas por elas à palavra dentista são "medo", "dor", "vontade de correr da sala [do dentista]", "achei ruim obturar dente por causa da dor, aquela máquina [o motor, a broca] é muito ruim", "medo da anestesia”, "medo de carão”, "pavor", “medo da agulha", "de extrair dentes", "fico tremendo", "morro de medo", "aquela posição [da cadeira] deitada dá mais medo ainda", "medo da força, ele é capaz de arrancar a boca toda".

Tais reações estão relacionadas à desconfiança na conduta adotada pelo dentista ou ao comportamento do mesmo "...tem pavor, medo, medo e quando você chega no consultório você vê o dentista de cara amarrada...".

Além da dor de dente enfrentada pela agudização de processos inflamatórios e resultantes do acúmulo de necessidade de tratamento sempre adiado, a expectativa das gestantes ao procurar o dentista é a de sentirem mais dor ainda “...quando vai puxar o alicate, dói”.
No decorrer dos encontros com os grupos, os medos com suas inúmeras faces foram sendo revelados pelas expressões dos rostos, pelas manifestações de rejeição a um conjunto de situações que as gestantes não querem enfrentar. Até mesmo pelas risadas elas denunciaram o nervosismo ao tratar do assunto.

"Medo dos carões, carões... eles não sabem nada o que a gente passa e já vai dizendo coisa". A atitude de submissão observada nas falas de algumas usuárias é um forte indicador da desigualdade social existente entre os dois participantes desse processo. De acordo com Freeman 2, tais desigualdades sociais dificultam a relação do dentista com o paciente, que deveria ser estabelecida entre dois adultos com o mesmo nível de igualdade no contexto de um real relacionamento, ou mesmo de um tratamento de aliança.

"O dentista é bonito, os dentes tudo bonito e a gente com os dentes tudo estragado". Outra forma de revelar as diferenças existentes e a distância entre dentista e paciente se expressa na vergonha que elas sentem pela condição de saúde bucal apresentada, em confronto com a condição do dentista que a atende, provavelmente resultante das largas desigualdades sociais verificadas no país, circunstância bem característica da população pesquisada neste estudo.

\section{- Custos}

A questão dos custos e a dificuldade que representam para a clientela foram espontaneamente suscitadas pelas gestantes no Cabo de Santo Agostinho, mesmo não tendo sido contemplada pelas perguntas dirigidas aos grupos focais trabalhados. As gestantes participantes dos grupos focais citaram o alto custo financeiro do tratamento odontológico privado e não dispor de plano de saúde como barreiras ao atendimento odontológico. A literatura científica referente ao tema tem apontado tais barreiras, como se encontra em Finch et al. 20, Wilson et al. 37, Mangskau \& Arrindell 18 e Tomar et al. 32 .

A população estudada no Cabo de Santo Agostinho apresenta uma porcentagem de 81,8\% sobrevivendo com até dois salários mínimos (U\$180 a preços de maio de 2001), o que indica acesso majoritário ao atendimento odontológico público por meio do Sistema Único de Saúde, que oferece acesso gratuito para o grupo pesquisado.

Em certos momentos da conversação com os grupos focais, algumas gestantes levantaram a possibilidade de buscar o atendimento em plano de saúde ou em consultório particular para fugir às barreiras previsíveis de serem 
encontradas no serviço público. Na maioria das vezes, elas cogitavam essa possibilidade como um sonho inatingível, em outras, como uma opção à reduzida oferta de serviços e de procedimentos oferecidos pelas unidades de saúde. "A pessoa vai no Posto porque não tem condições financeiras de ir para o [dentista] particular" ou "... [no particular] num dia só, com dinheiro, resolve o problema [da dor], se esperar fica sofrendo".

A partir daí as falas evidenciam algumas contradições. Por um lado as agentes comunitárias de saúde (ACS) reconhecem a dificuldade de acesso representada principalmente pela escassez dos serviços públicos oferecidos, por outro, em outra opinião, se contrapõem às colocações das demais gestantes e em defesa dos seus colegas do Posto “...até porque os dentistas do SUS, eles não atendem mal, esses daí [do Posto onde ela trabalha] são ótimos. A dificuldade é você conseguir chegar até eles".

Contraditoriamente, quando falam de sua própria situação ao enfrentar o atendimento gratuito, as ACS colocam a questão financeira como uma barreira a uma prestação de serviço mais ajustado às necessidades trazidas pelo paciente: "O INAMPS [lugar onde ela extraiu todos os dentes superiores] é para o povo, não é como no particular, que ali você está pagando um dinheiro vivo e é um dinheiro ali, que eles recebem bem, uma grana melhor do que pelo INAMPS. O salário [no INAMPS], acho que não é muito legal, muita gente ali, [os dentistas] arrancavam mais do que obturavam".

\section{- Dificuldade de acesso}

Os depoimentos das gestantes apontam a existência de dificuldades de agendamento, de enfrentar os riscos de sair de casa para marcar consulta, de transporte, de dinheiro para o transporte, de fazer perguntas em novos e desconhecidos ambientes, de ser atendida porque o dentista desaconselha o tratamento durante a gravidez, falta de tempo, tempo tomado ao trabalho remunerado, pouca credibilidade nos procedimentos efetuados e no diagnóstico do dentista, localização do consultório, absenteísmo do dentista, desconhecimento por parte das gestantes da existência do serviço gratuito e falta de informação.

Entre os fatores que figuram como barreiras ao atendimento está a dificuldade de acesso em todos os níveis, amplamente discutida na literatura por Blinkhorn et al. 24, Finch et al. 20, Rogers 17, Bernd et al. 36, Wilson et al. 37, Pavi et al. 12, Mangskau \& Arrindell 18, Jandrey \& Drehmer 33, Lathi et al. 34 e Nuto \& Nations 35.
A mudança na rotina diária, o fato de não ter com quem deixar os filhos menores, representaram barreiras para as gestantes estudadas que, somadas a outras pré-existentes, agravaram ainda mais a dificuldade de acesso aos serviços odontológicos.

Exemplo disso é a dificuldade causada pela insuficiência de políticas públicas que ofereçam suporte social como creches. "Saí de casa seis horas da manhã... meu marido está na maré e eu estou muito preocupada... os meninos ficaram só, saí tão cedo ... de manhã tem muita coisa pra fazer [afazeres domésticos]"

A falta de dinheiro para o transporte até o serviço odontológico é outra dificuldade de acesso que reflete a privação material enfrentada pelas gestantes e demonstra na prática a relação existente entre renda e saúde: “..eu não digo que vou [ao dentista] por causa do dinheiro da passagem, o negócio é que a gente não tem um dinheiro [salário] certo".

A vergonha de pedir informação em novos e desconhecidos ambientes, expressa também a sua condição social, o sentimento de ignorância, de mostrar pouca instrução: “...indo em grupo não tem vergonha de se informar... vergonha de chegar lá".

A opinião das gestantes (que são também agentes comunitárias de saúde) quanto à falta de informação é contrastada com a consciência repentina de sua postura, que se mostra semelhante àquela encontrada nas pessoas sob sua responsabilidade, e que esperam receber dos outros sem fazer um movimento em busca de solução para os próprios problemas: “...existe falta de informação... eu sou agente de saúde ... devia levar tudo para a comunidade sobre serviço de saúde, não utilizei, imagine o resto, não é?” E ainda: “...e também, sei lá, falta de informação... eu também poderia ter perguntado a alguém, eu tô cobrando igual às pessoas da comunidade. Eles querem tudo e não param pra perguntar 'como é que acontece' [como conseguir o atendimento]?".

"Tem que sair de madrugada [para conseguir a ficha para serem atendidas], faz medo...é perigoso passar.. tem maloqueiro maconheiro". Essa barreira inédita, identificada nos grupos focais do Cabo de Santo Agostinho, levanta a questão da segurança e acrescenta um obstáculo a mais às dificuldades de acesso pelo temor de enfrentar os riscos de sair de casa de madrugada para conseguir o atendimento. Condição presente nessa clientela que reside, invariavelmente, na periferia das cidades.

No Brasil, segundo dados do Instituto de Pesquisa Econômica Aplicada 38, 14,5\% da população vive abaixo da linha de pobreza. Estu- 
dos da Fundação Instituto Brasileiro de Geografia e Estatística 39 revelam proporção desigual no que se refere ao crescimento médio populacional, $1,63 \%$, e o que se verifica em algumas favelas é de $7,93 \%$. Os resultados dessa conjuntura são os altos níveis de violência manifestados contra a própria vizinhança. Em Pernambuco é de $74,7 \%$ a proporção de mortes por causas externas dos jovens entre 15 e 19 $\operatorname{anos} 40$.

A visão apresentada pelas gestantes desvenda a estreita relação existente entre saúde e determinantes sociais, representados por trabalho, renda, transporte, ambiente, educação, cultura, moradia. Tão abrangente concepção de saúde, resultante da 8a Conferência Nacional de Saúde 41, aparentemente não encontrou ainda espaço suficiente na agenda de planejadores em odontologia, nem sua plena aplicação por parte dos prestadores de serviço direto à população.

\section{Conclusões}

As conclusões deste estudo salientam questões importantes a serem consideradas na procura e na oferta dos serviços em saúde, especialmente, os de Odontologia. A abordagem qualitativa favoreceu a identificação de inúmeras situações que se configuram como barreiras ao atendimento odontológico. Assim, este estudo permitiu as seguintes conclusões em relação às gestantes do grupo estudado.

As crenças populares desaconselham gestantes a procurar o atendimento odontológico durante a gravidez, porque, segundo elas, existem riscos ao tomar a anestesia dental, perigos de hemorragia e perigos para o bebê. Elas se baseiam também na crença que a dor de dente está associada à condição da gravidez.

O sentimento mais forte em relação à própria saúde bucal e que as gestantes expressam em relação ao dentista é medo. Por essa razão, o que determina a procura de atendimento odontológico para as gestantes é uma dor de dente forte e contínua. Mesmo assim, sua perspectiva é a de sofrer mais ainda pelo medo de extrair o dente inflamado e vir a sentir dor. É possível que o medo revelado pelas gestantes esteja associado ao fato da população adulta representar na maioria das vezes o perfil mutilador da Odontologia. Dados de estudos epidemiológicos brasileiros demonstram que o CPOD (número de dentes cariados, perdidos e obturados) médio na idade adulta é de 22,5 , sendo que o componente perdido representa $67,5 \%$ dos dentes 42 .

A imagem dos dentistas, fortemente associada a autoritarismo, gera descrédito no diagnóstico e nos procedimentos realizados. As barreiras que dificultam o atendimento odontológico e que dizem respeito ao indivíduo são construídas com base em crenças, do relacionamento com o dentista e do modo como estão organizados os serviços de saúde.

Ao relatar a dificuldade representada pelos riscos de sair de casa de madrugada para marcar a consulta odontológica, a gestante se reporta a uma conseqüência da questão social que se reflete na violência urbana presente nas grandes cidades e enfrentada por ela em seu cotidiano, a partir da sua própria vizinhança, nas periferias onde reside. Esse aspecto das barreiras ao atendimento odontológico não foi mencionado em outros estudos.

A gestante se envergonha de fazer as perguntas necessárias no ambiente novo e desconhecido do consultório, teme as reprimendas dos dentistas pela sua condição de saúde bucal e compara a sua circunstância de vida com a classe social do dentista. Este conjunto de características são indicadores das condições sociais da população estudada e da distância entre ela e os dentistas que a atende.

Embora observando a cautela com que os achados deste estudo devam ser considerados, por se tratar de amostra não probabilística, o desenho apropriado do estudo possibilitou desvendar com clareza alguns pontos a ser melhorados na relação dentista-paciente, no planejamento e na prestação de serviços em saúde, na necessidade de educação continuada dirigida aos profissionais em exercício, na ênfase à humanização do atendimento começando pela formação de recursos humanos nas universidades, nos cursos profissionalizantes (THD e ACD) e na capacitação de pessoal contratado para o trabalho em saúde. 


\section{Resumo}

Este artigo enfoca, numa abordagem qualitativa, $o$ problema das barreiras ao atendimento odontológico de gestantes inscritas no Programa Saúde da Família no Cabo de Santo Agostinho, Pernambuco, Brasil. Para tal, trabalhou-se com três grupos focais compostos por quatro a nove gestantes. Os dados foram analisados usando a técnica de análise de conteúdo. As principais barreiras concernentes ao indivíduo observadas foram: crenças populares que desaconselham a busca do atendimento odontológico na gravidez, baixa percepção de necessidade e medo de sentir dor. Além disso, as gestantes relataram a dificuldade de sair de casa de madrugada para marcar a consulta, salientando a questão social da violência urbana, um aspecto das barreiras ao serviço odontológico que não foi mencionado anteriormente na literatura. O artigo conclui apontando a importância da educação em saúde para as usuárias gestantes, da humanização do atendimento e da educação continuada dirigida aos profissionais em exercício.

Mulheres Grávidas; Programa Saúde da Família; Serviços de Saúde Bucal

\section{Colaboradores}

O. M. R. Albuquerque foi responsável pela versão preliminar do artigo. Todas as autoras participaram das etapas seguintes do trabalho. A editoração final do artigo foi realizada por C. Abegg.

\section{Referências}

1. Fundação Instituto Brasileiro de Geografia e Estatística. Pesquisa Nacional por Amostragem de Domicílio. http://www.ibge.gov.br (acessado em 10/Ago/2000).

2. Freeman R. Barriers to accessing and accepting dental care. Br Dent J 1999; 187:81-4.

3. Freidson E, Feldman JJ. The public looks at dental care. J Am Dent Assoc 1958; 57:3.

4. Kegeles SS. Why and how people use dental service. Int Dent J 1974; 24:347-51.

5. Gale EN. Fears of dental situation. J Dent Res 1972; 51:964-6.

6. Kleinknecht RA, Klepac RK, Alexander LD. Origins and characteristics of fear of dentistry. J Am Dent Assoc 1973; 86:842-7.

7. Cohen LK. Converting unmet need for care to effective demand. Int Dent J 1987; 37:114-6.

8. Sheiham A. Is there a scientific basis for six monthly dental examination? Lancet 1977; 2:442-4.

9. Eddie S. Frequency of attendance in the General Dental Service in Scotland: comparison with claimed attendance. Br Dent J 1984; 155:363-6.

10. Elderton RJ. Routine six monthly checks for dental disease. Br Dent J 1985; 9:277-8.

11. Elderton RJ. Six-monthly examinations for dental caries. Br Dent J 1985; 5:370-4.

12. Pavi E, Kay EJ, Stephen KW. The effect of social and personal factors on the utilization of Dental Services in Glasgow, Scotland. Community Dent Health 1995; 12:208-15.

13. Löe H. Periodontal changes in pregnancy. J Periodontol 1965; 36:209-17.

14. Chiodo GT, Rosenstein DI. Dental treatment during pregnancy: a preventive approach. J Am Dent Assoc 1985; 110:365-8.

15. Offenbacher S, Katz V, Fertik G, Collins J, Boyd D, Maynor G, et al. Periodontal infection as a possible risk factor preterm low birth weight. J Periodontol 1996; 67:1103-13.

16. Nuammah I, Annan BDRT. Periodontal status and oral hygiene practices of pregnant and non-pregnant women. East Afr Med J 1998; 75:712-4.

17. Rogers SN. Dental attendance in a sample of pregnant woman in Birmingham. Community Dent Health 1991; 8:361-8.

18. Mangskau KA, Arrindell B. Pregnancy and oral health: utilization of oral health care system by pregnant women in North Dakota. Northwest Dent 1996; 75:23-8.

19. Albuquerque OMR, Santos M, Rodrigues CS. Avaliação do sistema de agendamento de consultas odontológicas do Programa Saúde da Mulher no Cabo de Santo Agostinho - PE. In: XVI ENATESPO: XVI Encontro Nacional de Administradores e Técnicos dos Serviços Públicos Odontológicos; 2000 Set 6-10; Brasília. Brasília: Editora UnB; 2000. p. 74-5.

20. Finch H, Keegan J, Ward K, Sen BS. Barriers to the receipt of dental care: a qualitative study. London: Social and Community Planning Research; 1988.

21. Blinkhorn AS, Leathar DS, Kay JK. An assessment of the value of qualitative and quantitative data collection techniques in oral health promotion 
research. London: British Dental Association Headquarters; 1992.

22. Nettleton S. Understanding dental health beliefs: introduction to ethnography. Br Dent J 1986; 161:145-7.

23. Gift HC. Values of select qualitative methods for research, education and policy. J Dent Educ 1996; 60:703-8.

24. Blinkhorn AS, Hastings GB, Leathar DS. Attitudes towards dental care among young people in Scotland. Br Dent J 1983; 5:311-3.

25. Redford M, Gift HC. Dentist-patient interations in treatment decision making: a qualitative study. J Dent Educ 1997; 61:16-21.

26. Krueger RA. Focus groups. Thousand Oaks: Sage Publications; 1988.

27. Glaser BG, Anselm AL. The discovery of grounded theory: strategies for qualitative research. New York: Aldine Gruyter; 1967.

28. Frankfort-Nachmias C, Nachmias D. Research methods in the social sciences. London: Biddles 1992.

29. Pope C, Mays N. Qualitative research: rigour and qualitative research. Br Med J 1995; 311:109-12.

30. Bardin L. Análise de conteúdo. Lisboa: Edições 70; 1979.

31. Programa das Nações Unidas para o Desenvolvimento. Atlas do desenvolvimento humano no Brasil. Desenvolvimento humano, condições de vida [CD-ROM]. Brasília: Programa das Nações Unidas para o Desenvolvimento/Instituto de Pesquisa Econômica Aplicada/Fundação João Pinheiro/Fundação Instituto Brasileiro de Geografia e Estatística; 1998.

32. Tomar SL, Azevedo AB, Lawson R. Adult dental visits in California: success and challenges. J Public Health Dent 1998; 58:275-80.

33. Jandrey CM. Absenteísmo no atendimento clínico-odontológico: o caso do Módulo de Serviço Comunitário (MSC) do Centro de Pesquisas em
Odontologia Social (CPOS) [Dissertação de Mestrado]. Porto Alegre: Programa de Pós-graduação em Odontologia, Universidade Federal do Rio Grande do Sul; 1999.

34. Lahti SM, Hannu WH, Vaskilampi T. The perceptions of users about barriers to the use of free systematic oral care among finish pre-school children - a qualitative study. Acta Odontol Scand 1999; 57:139-43.

35. Nuto S. Avaliação cultural do serviço de saúde de Beberibe - CE: a vez e a voz do usuário. [Dissertação de Mestrado]. Fortaleza: Universidade Federal do Ceará; 1999.

36. Bernd B, Souza CB, Lopes CB, Pires Filho FM, Lisbôa IC, Curra LCD, et al. A percepção popular sobre saúde bucal: o caso das gestantes do Valão. Saúde em Debate 1992; 34:339.

37. Wilson MC, Holloway PJ, Sarll DW. Barriers to the provision of complex dental treatment for dentate older people: a comparison of dentists' and patients' views. Br Med J 1994; 177:130-4.

38. Instituto de Pesquisa Econômica Aplicada. Data, temas, fontes. http://www.ipea.gov.br (acessado em 21/Set/2001)

39. Fundação Instituto Brasileiro de Geografia e Estatística Censo 2000. Notícias. http://www.ibge. gov.br (acessado em 10/Ago/2000).

40. Fundação Instituto Brasileiro de Geografia e Estatística. Censo 2000. Press release number 061. http://www.ibge.gov.br (acessado em 10/Ago/ 2000).

42. Bellini H, Pinto VG. Delivery of oral health care and implications for future planning. In: Pine CM, editor. Community oral health. Oxford: Wright; 1997. p. 291-7.

41. Ministério da Saúde. 8ạ Conferência Nacional de Saúde. Brasília: Ministério da Saúde; 1986.

Recebido em 20/Mai/2003

Versão final reapresentada em 31/Out/2003 Aprovado em 26/Nov/2003 